\title{
LA INVESTIGACION: \\ Una alternativa para la formación y el desempeño profesional
}

Por: Nelly T. Martínez M.*

\section{Introducción}

La experiencia de varios años alrededor de la enseñanza de la investigación en pregrado y especialización, los avances conceptuales logrados posteriormente a las tesis de maestría en Educación para salud ${ }^{1}$ y la constante búsqueda bibliográfica sobre la pedagogía como disciplina articuladora del saber en educación física, me permitieron encontrar parte de la respuesta a la pregunta que por varios años había formulado: ¿Cómo hacer para que el estudiante y el profesional tengan una mejor formación y desempeño?

A partir de este cuestionamiento y después de muchas búsquedas bibliográficas, de planteamiento de tesis muy globalizantes, he llegado a la elaboración de este ensayo que pretende mostrar, de una parte, la importancia de una formación centrada en el dominio de los fundamentos científicos, pedagógicos y humanos, como elementos básicos y, por otra parte, el fortalecimiento del status profesional que debe distinguir al profesor de educación física, de otras ocupaciones u oficios similares, a partir de la consolidación de un pensamiento reflexivo y una actitud investigativa que haga posible el ejercicio de la docencia caracterizada por una constante renovación y cualificación.

Para nadie es una novedad que el deseo de saber encontrar la verdad y las respuestas a la realidad, no siempre ha encontrado circunstancias propicias para su desarrollo. Tampoco lo es que la investigación es el camino para la ciencia y, menos aún, que la producción de conocimiento se ha constituido en el interés fundamental para el desarrollo económico, porque el motivo de la lucha en este aspecto, ya no es la teoría; se ha hecho realidad el enunciado de Francis Bacon: "El conocimiento es poder"2.

De lo anterior, queda claro que la producción de conocimiento siempre ha sido necesaria, y hoy más que nunca, particularmente en el campo de las disciplinas o ciencias de la Educación Física y el Deporte, pues las grandes preguntas que hoy se plantean alrededor de su naturaleza científica son: “¿...Es ciencia? ¿En qué medida y cuál es su naturaleza?"3. Esto hace necesario no sólo el debate sobre su objeto de estudio, sino la reflexión sobre la formación profesional en estos campos, lo que nos remite a la investigación como alternativa.

Pero, ¿cómo asumir la investigación? En primer lugar, habría que aceptar que la investigación es el camino para la ciencia, pero no siempre que se investiga se produce conocimiento de tal calidad que pueda articularse a la ciencia formal y legítima en un momento y lugar determinados.

\footnotetext{
${ }^{*}$ Profesora Facultad de Educación Física de la U.P.N.

${ }^{1}$ La tesis se tituló Papel del profesor de la Educación Física en la Promoción de la salud. Expectativas y realizaciones UPN 1986.

${ }^{2}$ BORRENAU, Franz. La coyuntura de Descartes En Argumentos 30. Pags. 40.199

${ }^{3}$ RODRÍGUEZ LÓPERZ, Juan. Deporte y ciencia. Editorial INDE, Barcelona España 1995. Pag. 207. 
En segundo lugar no se puede pasar por alto que, pese a las diversas concepciones que pueden hacerse sobre ella, la investigación es, ante todo, búsqueda de conocimiento, de un conocimiento para el hombre que lo acerque a la vida; pues ésta no puede concebirse sin mirarla, consentirla, conservarla. Por tanto, la actividad investigativa requiere ser incorporada como una actitud de vida que se constituye a través de un largo camino, en el transcurso del cual se descubre el conocimiento que esconden las cosas. Pero indudablemente requiere de muchas andanzas y más en Educación Física que tiene mucho por aclarar y recorrer Si se acepta que la investigación tiene niveles, secuencias y profundidades, entonces podremos atrevemos a adelantar recorridos sencillos como los que se generan a partir de una actitud investigativa.

\section{La investigación como actitud en la formación y el desempeño profesional docente.}

Ya se planteó que, la investigación es una alternativa de superación y mejoramiento profesional, si se construye como una actitud de vida, desde el marco de la formación universitaria, y se ejecuta desde el contexto del desempeño, cualquiera sea el rol o ubicación del Educador Físico.

La actitud, en un sentido general, puede entenderse como disposición, querer hacer, potencia para actuar Nótese que es disposición para el acto y no el acto mismo, pues muchas veces, se obra en aparente o real oposición a nuestra disposición o actitud. Aquí cabría preguntarse, por ejemplo: ¿Cuál es nuestra actitud frente a la profesión de educador?, ¿Se tiene la disposición, el gusto, el deseo por la superación y el enriquecimiento de la misma?

Ahora bien, la actitud se mueve entre dos polos: positivo y negativo. Esto significa que las actitudes son disposiciones a favor o en contra de algo o alguien, situación o evento, y no asume valores absolutos, sino que se mueve entre los extremos, lo cual quiere decir que presenta términos medios y, por lo tanto, puede mejorarse o retrocederse en ella.

Por otra parte, la actitud, como disposición de la persona, está constituida por tres componentes: el cognoscitivo, el afectivo y el de la acción, que interactúan dinámicamente entre sí, aunque no siempre en relación directamente proporcional. En consecuencia, la actitud hacía la profesión de educador físico, puede definirse inicialmente como el conjunto de creencias, saberes, sentimientos y disposiciones para actuar en busca de la verdad disciplinar; curiosidad insaciable, deseo permanente por la transformación sistemática de la realidad, por el conocimiento del movimiento y corporalidad humanas o de la actividad física y lúdica, como mediación educativa y posibilidad de transformación social y cultural.

Profundizando un poco más, y teniendo en cuenta sus tres aspectos constitutivos, la actitud investigativa hacia la profesión de educador físico, se debe reforzar de la siguiente manera:

- Desde lo cognoscitivo. Propiciar la consolidación de un acervo de conocimientos, creencias, ideas, conceptos, teorías y formulaciones sobre la investigación, la pedagogía, la educación, la cultura, las ciencias del deporte y la educación física, durante la formación universitaria y/o reformulándolos desde la realidad sensible y cotidiana en que se ejerce la profesión.

- Desde lo afectivo. Crear condiciones y fortalecer situaciones de la vida sentimental y emocional. Así: el gusto, el placer, el amor, la empatía, la pasión, la alegría por la 
observación consciente, la problematización, la búsqueda de soluciones, la explicación y la teorización, la crítica permanente, la lectura constante, la necesidad de transformación, la discusión compartida, la fundamentación, pero, sobre todo, el gusto por la duda, el cuestionamiento y el conocimiento, no sólo en lo relacionado con los fundamentos específicos de la carrera, sino del entorno en que se construye la cultura del movimiento, del cuerpo, del deporte. El gusto por la reflexión sobre la enseñanza es algo que no puede faltar en la estructuración de esta dimensión de la actitud.

- Desde lo conativo o plano de la acción. La actitud investigativa se incrementa potenciando la disposición para actuar e intervenir en los procesos que preparan científica y pedagógicamente al profesional de la educación física, tanto a nivel teórico, como práctico. Disposición para la enseñanza reflexiva, para el cuestionamiento educativo, deportivo y cultural. Tendencia a liderar procesos organizativos para la superación de barreras sociales y cognitivas; fuerza interior para actuar transformadora y constructivamente sobre la profesión y el entorno en general.

Las actitudes no nacen espontáneamente ni son positivas o negativas por antonomasia. Como toda característica social, se construyen en un contexto, se aprenden a través de la formación y la experiencia y pueden ser específicas y evidenciarse en la conducta, en el comportamiento, en las relaciones e interacciones en las que operan las normas y en las que el profesional o estudiante de educación física, deporte, o recreación, dirigente deportivo o entrenador, se ven influenciados y determinados por dos microentornos: El universitario, o de formación profesional, y el específico del desempeño laboral.

Por otra parte, cabe señalar que si se tiene en cuenta que tanto la Universidad como el entorno del desempeño son los espacios, por excelencia, de conocimiento y construcción de los estudiantes y profesionales que lideran el desarrollo de un país, no cabe la menor duda de que éstos ámbitos constituyen el campus propicio, la tierra adecuada para el cultivo de la actitud investigativa, así como lo es también toda la escolaridad. En este sentido, es claro, que el currículo debe ser considerado como proyecto y espacio de investigación, pero a su vez, estar rodeado por un clima organizacional y académico en el que se dinamicen y propicien condiciones para las prácticas investigativas; se cultive, se valore y se privilegie, en el quehacer, el espíritu científico desde todos los ámbitos de la formación, y en especial, desde el pedagógico, el cual ha de posibilitar el pensamiento crítico y reflexivo para la construcción y reconstrucción, transformación y apropiación del saber y del conocimiento de los hechos, de la cultura física y deportiva como elemento esencial en la formación de una actitud científica.

En estas circunstancias, la reflexión no puede ser asumida a la ligera como un simple cuestionar o pensar, sin saber alrededor de qué o por qué, pues no sólo supone un proceso sino que, por otra parte, supone unas relaciones pedagógicas diferentes a las de la transmisión y autoritarismo.

\section{El pensamiento reflexivo: una posibilidad desde la Pedagogía del Portento.}

Además del reforzamiento de la actitud, a partir de sus tres componentes, una segunda condición o requerimiento para la formación de la actitud investigativa está relacionada con el pensamiento reflexivo, como proceso intencionado, desde una práctica pedagógica posibilitadora. En este sentido, es necesario hacer algunas precisiones.

En los últimos 15 años han surgido denominaciones para referirse al profesor o profesional de la actividad física. El tradicional docente se ha querido reivindicar por el 
de "maestro," "orientador," "amigo del estudiante". Particularmente este último es muy frecuente en el campo de la actividad física y deportiva, pero no es tanto el cambio de denominación, sino la forma de asumir el quehacer, lo que hará redimensionar su papel en la sociedad. Pero, ¿cuál es la ciencia con la que el estudiante conseguirá ser un experto profesional de la actividad Física y el Deporte? "Sin lugar a dudas, es la pedagogía..., el educador físico es un pedagogo de las actividades relacionadas con el movimiento humano, con el ejercicio físico y el deporte"4.

Pero, ¿qué significa ser un pedagogo? La pregunta nos remite inmediatamente a la pedagogía, sobre la que se han planteado muchas concepciones, que van desde su similitud, con la didáctica "como técnicas destinadas a dirigir la enseñanza", hasta considerarla como la Teoría de la Educación.

En forma muy genérica, pero muy profunda y universal, pienso al igual que José Muñoz, que la pedagogía "es una forma de asumir las interacciones que se nos presentan en cualquier entorno. La pedagogía es una actitud frente al mundo, una forma de encararlo, de asirlo, de comprenderlo; la pedagogía es, por tanto, un acto valorativo: significa toma de posición ideológica". Sin embargo, como estado de relación con el otro, y con lo otro, se puede asumir desde "El portento o del ser altivo" en oposición a "Lo indubitable o del ser de mansedumbre",

Desde la primera dimensión, el ser altivo o del portento, todos los hechos que rodean al hombre, al estudiante, al profesional, son un estímulo, un motivo para investigar, sin interesar si sobre esto o aquello hay ya mucha o poca teoría o muchas verdades o incertidumbres. Es con una actitud pedagógica del ser altivo que la investigación se asume como principio, como volver a empezar, como volver a recorrer lo ya andado. En esta dimensión pedagógica las verdades son relativas, el saber es un motivo de placer y la investigación se ubica como un principio; es decir, como el fundamento iniciador de cualquier interacción del estudiante o del profesor, ya sea con el saber o con el entorno.

Desde la dimensión pedagógica de lo indubitable o del ser sumiso, la investigación no es posible; la verdad se asume como dada y la disertación del profesor como dogma, como autoridad. Los programas curriculares están ya definidos, prefijados su orden y sus secuencias; las metodologías de investigación son esquemas, limitaciones, guías forzadas.

En la pedagogía del ser altivo, la investigación siempre es posible porque crea lazos de comprensión con las posibilidades del otro, porque crea espacios y oportunidades, porque no confunde lo humano con lo natural. La pedagogía del portento conoce los deslindes de las disciplinas y sus aportes dentro de las Ciencias del deporte, pero no se subyuga ante la Biología, la Sicología, o la Historiografía. En la pedagogía del protento, la investigación se presenta bajo una mirada interdisciplinar, siempre posible.

En la pedagogía de la sumisión, el estudiante o profesional de la educación física le teme a aventurarse en otro tipo de búsquedas que rompan los cientificismos construidos, especialmente con la mirada de los criterios naturales y de la física. Para él, en el ser indubitable o de mansedumbre no hay cuestionamiento permanente, ni duda, se asume y subsume lo que se trae de afuera, lo que se produce en otros contextos.

\footnotetext{
${ }^{4}$ RODRÍGUEZ LÓPEZ, Juan. Deporte y ciencia. Editorial INDE. Barcelona España 1995. Pag. 207.

${ }^{5}$ NERICI, IMIDEO, Hacia una didáctica general dinámica. kapeluz, Buenos Aires 1973, pag. 53.

${ }^{6}$ MUÑOZ, José Arturo. Aproximación crítica a la pedagogía. Cmprodic. 
Ahora bien, como la Pedagogía también puede entenderse como teoría de la Educación y la acción humana, "como la disciplina que conceptualiza, aplica y experimenta los conocimientos referentes a la enseñanza de los saberes específicos", esto nos lleva a concluir que el proceso reflexivo, como parte fundamental de la formación investigativa y pedagógica, requiere la elaboración de una teoría que pueda orientar el quehacer del docente a nivel práctico. Así, la pedagogía puede definirse como el conjunto de saberes y constructos elaborados sobre y a partir de la práctica educativa, o como la acción humana, a través de un proceso de acción-reflexión-contrastación-decantación y, nuevamente, aplicación o acción de la enseñanza; entendida ésta como opción del pensamiento y no como proceso de transmisión.

Si la pedagogía gira alrededor de la enseñanza, supone unas categorías fundamentales; el maestro, el estudiante, el conocimiento, el saber, el método, los recursos, las finalidades, el entorno..., y otras que se consideren esencia y eje dinamizador de la construcción pedagógica y el inicio de la teoría pedagógica de la educación física. Si el profesor de educación física entra por las vías del pensamiento reflexivo, indudablemente se inicia en el camino de la investigación científica, pues éste proceso lo lleva a adquirir las características que debe reunir un científico y que en la práctica son:

- Disciplina (pensar ordenadamente sobre algo).

- Observación sistemática - regularizada - constante.

- Capacidad para el análisis y la identificación de problemas.

- Búsqueda de explicaciones (hipótesis).

- Elaboración de teorías (abstracciones generalizantes para su microentorno).

- Réplica de los procesos exitosos (no por ello verdades universales).

\section{¿Qué requiere el pensamiento reflexivo?}

De acuerdo con lo dicho, la reflexión exige, antes que todo, de una postura pedagógica abierta. En el campo de la educación física-deportiva o recreativa, siempre es posible una dimensión pedagógica de alteridad; sin embargo, tratándose del rendimiento, del entrenamiento, de la dirección o de la gestión deportiva, la interacción pedagógica tiende a ser del tipo indubitable, pero no necesariamente impide la reflexión para la transformación del establecimiento. Lo que se supone, entonces, es articular en la práctica el espíritu investigativo, antes que hacer oficiantes o investigadores, situación que es loable, pero no prioritaria. En consecuencia, se debe buscar que el pedagogo, estudiante o profesional en este campo, haga de su práctica cotidiana un espacio investigativo que le permita transformarla progresivamente y transformarla creativamente, para enriquecer los procesos de formación, cualificar la profesión y consolidar la disciplina.

Para esta aspiración es necesaria una exigencia: que el educador físico, desde su proceso formativo, sea preparado, habilitado en el pensamiento reflexivo, para que, sabiendo en qué consiste y cómo se hace, pueda ejercerlo fuera de la universidad. Porque es necesario que someta a reflexión sus conocimientos, las creencias que ha construido y las prácticas que ha introyectado.

La imagen del profesional de la actividad física, como un pedagogo reflexivo, se enmarca en la visión de la enseñanza, como una opción del pensamiento, que requiere de un conocimiento profundo del sujeto de la enseñanza (el estudiante), ubicado en un micro y macroentorno, todo lo cual debe ser articulado e interpretado, adaptado y aplicado 
según las contingencias particulares, con miras a cualificar la profesión y la disciplina. Con la reflexión, la investigación se consolida como el eje articulador del quehacer diario del maestro; se legitima por la práctica, la calidad de la enseñanza, y se logra la diferenciación de la práctica de sentido común, de la práctica científica y pedagógica.

\section{¿Qué es reflexión?}

A pesar de que el término reflexión es usado con frecuencia y su abuso ha llevado a un aparente acuerdo para incluirlo en muchos discursos de transformación de la acción del profesional de la educación física o deportiva, no existe, sin embargo, unanimidad en su definición. Aunque normalmente se asocia con mejoras, crecimiento, toma de conciencia, mediante la mirada crítica, no se llegan a analizar sus perspectivas. No obstante, que el profesor o el profesional, en general, la tienen como una meta, él es alguien que está dispuesto a reflexionar, y éste es el medio o fin.

Pero, ¿en qué consiste esa actividad reflexiva? Mckay y Marland, 1978 consideran, el término reflexión como una "categoría del pensamiento interactivo", significando que el profesor está pensando la lección de una manera diferente a como la realizó.

Calderhead, remite su concepción de reflexión, al origen de la expresión dentro del rol del profesor en sus cogniciones. Se remonta a la enseñanza reflexiva.

Hace referencia a expresiones tales como: "práctica reflexiva-formación del profesor orientada a la indagación, reflexión -en-la- acción; profesor como investigador, profesor como tomador de decisiones, profesor como profesional, y el profesor como resolutor de problemas".

Muchos autores se refieren a la concepción hecha por Donald Schon, (1983 - 1992), reflexión —en - la - acción y reflexión - sobre - la acción. Para este autor el corazón de la reflexión es la reconstrucción, pues ésta altera el modo en que son vistos los datos reales, al hacerlos presentes bajo una nueva perspectiva. Por eso cuando uno reflexiona asume que las estructuras no son estáticas sino que la mente se esfuerza por expandirlas, refinar-las y alternarlas, y si esto se presenta, la reconstrucción aparece. Se podría decir que una persona, un profesional o un pedagogo del movimiento está reflexionando cuando está dedicado a "estructurar" su percepción de la situación de enseñanza, de la evaluación, de la interacción o relación con el saber o con los estudiantes, o micro y macro entorno, y se dispone y se ocupa de modificar sus acciones o aprendizajes; a la construcción de la práctica no se llega sólo por la actitud reflexiva, ésta es solo una tendencia a alterar las estructuras de pensamiento (Actitud pedagógica).

Ahora bien, ¿cuáles son los dominios o categorías de reflexión para un profesional de la Educación Física que se desenvuelve como profesional pedagogo?

Las categorías están relacionadas, como se enunció anteriormente, con el maestro, el estudiante, el saber, los procesos, el entorno y todas aquellas variables que giran alrededor de la enseñanza, la dimensión curricular y la gestión. Desde estas categorías, las preguntas que pueden ayudar a iniciar la reflexión, pueden ser:

- Para qué enseñar movimientos? (Ethos).

- ¿Qué enseñar en la clase que sea significativo para el estudiante en su contexto cultural y socio-económico?

- ¿Cómo enseñar y relacionarse para que en el otro se genere el ser altivo y no sumiso? 
Los temas o dimensiones de la reflexión pueden ir cambiando; sin embargo, es preciso tener en cuenta que la reflexión no sólo se dé sobre lo que "sale mal" o "vemos mal", sino también sobre lo que nos "sale bien". Además, puede hacerse durante o después de la planificación, o durante o después de la enseñanza o de la interacción.

Asimismo, dado que la acción reflexiva está compuesta por funciones claras, es necesario tenerlas en cuenta si se pretende realmente la reconstrucción de la práctica y la elaboración de teorías o al menos del saber sistematizado. Estas funciones son:

- Orientación: prever el rumbo de la acción que se va a reflexionar.

- Planificación: previsión ordenada de las secuencias y acciones alternativas.

- Dirección: gestión encaminada al logro.

- Comprobación: evaluación para verificar lo hecho.

- Interpretación: lectura de fondo, más allá de lo empírico, para develar nuevos significados de las acciones realizadas.

- Reconstrucción: retomar todo el proceso y precisar las nuevas formas en que se presenta la alteración, elaborar la construcción (saber) que se ha decantado.

La puesta en práctica de estas funciones garantiza un excelente comienzo para la reflexión; sin embargo, la complejidad o profundidad de la misma, depende del nivel en que ella se dé.

- Nivel Técnico: se refiere a la aplicación efectiva del conocimiento educativo, constituye el primer nivel.

- Nivel Educativo: es el segundo nivel de la acción reflexiva. Se refiere a la identificación de problemas de la interacción práctica, a partir de los referentes y perspectivas asumidas por el docente, derivadas de las valoraciones de las intervenciones educativas y desde los referentes teóricos poseídos.

- Nivel Ético: es el tercer nivel de la reflexión crítica. Incorpora criterios morales y éticos acerca de la acción práctica. Aquí, la reflexión se encamina a los fines de la educación. Ahora bien, para que estos procesos de reflexión realmente operen, es necesario que:

1. Los estudiantes aspirantes a pedagogos de la educación física, el deporte o la recreación, se formen y se entrenen en estos procesos; que logren la habilidad de fundamentar; es decir, de razonar sólidamente sobre su enseñanza, para actuar hábilmente.

2. El estudiante no sólo defina qué es el pensamiento reflexivo sino que sepa llevarlo a la práctica en un momento dado, en circunstancias y contextos especiales.

3. El futuro profesional adquiera capacidad para identificar problemas en su práctica, encuentre modos de solucionarlos y se disponga a aplicarlos.

4. El contexto de formación propicie el pensamiento reflexivo, la actitud y espíritu científico, esto es, que el ambiente de formación sea de la misma naturaleza, ya que el sujeto tiende a repetir los procesos en que se formó.

5. Se adquieran habilidades en las formas didácticas fundamentales:

- Observación analítica - focalizada — sistemática 
- Escritura de las observaciones hechas tanto a sí mismo como a los fenómenos; es decir, aprender a documentar lo que se hace, lo que se reflexiona.

Estas recomendaciones son sólo para el estudiante. El egresado debe tener claro que teniendo acceso a las dimensiones de la ciencia podrá ir consolidando las bases de su profesionalidad, pues éstas no se logran únicamente con títulos profesionales. Es necesario, por lo tanto, que el profesional se involucre, desde el inicio de su carrera, en los procesos de reflexión » en general, en toda la actividad científica a saber:

- Investigación: para la obtención de nuevos datos y conocimientos sobre ciencias fundantes y articuladoras como la pedagogía y la didáctica.

- Aplicación: aprovechamiento de los descubrimientos en las ciencias propias, auxiliares 0 anexas, en la solución de los problemas educativos, pedagógicos 0 didácticos.

- Sistematización: ordenación del conjunto de sus reflexiones pedagógicas, administrativas, de gerencia, etc, con el fin de ir creando el contexto o su práctica.

- Transmisión: de contenidos teóricos, de saberes, resultado de su propia reflexión, de experimentos hechos por otros, o de su práctica pedagógica investigativa.

Asimismo, cabe señalar que en el plano de la política es necesario no estar subordinado científicamente, y que es necesario educarse a sí mismo, regularse y autocontrolarse; aunque no siempre es así, pues las políticas de formación y desempeño investigativo de los pedagogos y profesionales del movimiento o actividad física están en abierta oposición con las condiciones reales del ejercicio profesional. Esta situación, generalmente lleva a preguntarse por la posibilidad de crear con-textos reales propios para la investigación y para la formación de los estudiantes. Sin embargo, esto no debe constituirse en obstáculo, por el contrario, es el reto que se debe asumir desde la Educación Física, pues se trata de redimensionalizar la profesión, de buscar posibilidades de educar científicamente a quienes son usuarios. Es urgente que la investigación sea el eje articulador del quehacer diario, se trata de legitimar, por la calidad, lo que se practica y enseña.

\section{Bibliografía}

MUÑOZ, José Arturo. (1998). Teoría crítica a la pedagogía. CORPRODIC, págs 18-24.

DE ZUBIRÍA, Julián. (1998). Tratado de pedagogía conceptual. Los modelos pedagógicos. Fundación Alberto Merani Fondo de Publicaciones Bernardo Herrera M., Bogotá,

RODRÍGUEZ LÓPEZ, Juan. (1995). Deporte y ciencia. Publicaciones INDE. Primera Edición 1995. Barcelona - España.

ZULUAGA, Olga Lucía. (1987). Pedagogía e historia. Ediciones Foro Nacional por Colombia. Primera Edición, pág. 21.

CORTAZAR, José Miguel. (1990). Los límites de la autonomía profesional. En: revista española, ANTHROPOS, págs 79-95. 
GALLEGO ARREFAT, María Jesús. (1994). Aportaciones conceptuales y estrategias para promover la reflexión del profesor sobre la práctica, con medios didácticos. Págs. 117-124. En: Revista Española de Pedagogía No. 197. Enero-Abril 1994. Granada España. 